\title{
Outcomes of Adding Induction Chemotherapy to Concurrent Chemotherapy for Nasopharyngeal Carcinoma Patients with Moderate-Risk in the Intensity-Modulated Radiotherapy Era
}

This article was published in the following Dove Press journal:

Therapeutics and Clinical Risk Management

\author{
Zhen Su ${ }^{1}$, \\ Guo-rong Zou',* \\ Jie Tang' \\ Xiu Yue $\mathrm{Li}^{\prime}$ \\ Fang-Yun $\mathrm{Xie}^{2}$ \\ 'Panyu Central Hospital, Cancer Institute \\ of Panyu, Guangzhou, People's Republic \\ of China; ${ }^{2}$ Department of Radiation \\ Oncology, Sun Yat-Sen University Cancer \\ Center, State Key Laboratory of \\ Oncology in South China, Collaborative \\ Innovation Center for Cancer Medicine, \\ Guangzhou 510060, People's Republic of \\ China
}

*These authors contributed equally to this work
Correspondence: Fang-Yun Xie Department of Radiation Oncology, Sun Yat-sen University Cancer Center; State Key Laboratory of Oncology in South China; Collaborative Innovation Center for Cancer Medicine, Guangzhou 510060, People's Republic of China Tel +862087343484

Email xiefy0758@sina.com
Background: The aim of this study was to evaluate the efficacy of induction chemotherapy (IC) in nasopharyngeal carcinoma (NPC) patients with moderate-risk treated with intensitymodulated radiotherapy (IMRT).

Methods: We retrospectively assessed 506 patients with T1-2N1M0 or T3-4N0-1M0 NPC (according to the 2010 UICC/AJCC staging system) who received concurrent chemoradiotherapy (CCRT) with or without IC at a single center in China between 2005 and 2010 Survival outcomes were compared between the IC + CCRT and CCRT groups using the Kaplan-Meier method, Log-rank test and a Cox regression model.

Results: Among the 506 patients, CCRT alone resulted in equivalent overall survival $(86.8 \%$ vs $88.5 \%, p=0.661$ ), progression-free survival ( $79.6 \%$ vs $79.6 \%, p=0.756)$, locoregional relapse-free survival $(90.2 \%$ vs $87.0 \%, p=0.364)$ and distant metastasis-free survival $(88.0 \%$ vs $89.8 \%, p=0.407)$ to IC plus CCRT. In multivariate analysis, IC did not lower the risk of death (HR 0.76, 95\% CI 0.46-1.25, $p=0.278$ ), progression (HR 0.78, 95\% CI 0.51-1.19, $p=0.244$ ), locoregional relapse (HR $1.06,95 \%$ CI $0.81-1.42, p=0.651$ ) or distant metastasis (HR $0.66,95 \%$ CI $0.38-1.15, p=0.140$ ) in the entire cohort; similar results were obtained in stratified analysis based on N category (N0 vs N1) and EBV DNA ( $<$ vs $\geq 4000$ copies $/ \mathrm{mL}$ ).

Conclusion: Addition of IC to CCRT does not improve survival outcomes in moderate-risk NPC; the use of IC should be carefully considered in these patients, though additional prospective trials are warranted to confirm the conclusions of this study.

Keywords: nasopharyngeal carcinoma, induction chemotherapy, prognosis, survival

\section{Introduction}

Nasopharyngeal carcinoma (NPC) is endemic throughout South China, Southeastern Asia, and North Africa, ${ }^{1}$ with an annual incidence of approximately 15-30 per 100,000 in Southern China. ${ }^{2}$ The primary treatment modality for NPC is radiotherapy. Concurrent chemoradiotherapy (CCRT) is recommended as the main treatment for locoregionally advanced NPC in the National Comprehensive Cancer Network (NCCN) guidelines. ${ }^{3}$

Distant metastasis is now the predominant failure pattern. ${ }^{4}$ In an attempt to reduce the rate of distant metastasis and further improve OS, several groups have explored different strategies in the IMRT era, such as adding induction chemotherapy (IC) or adjuvant chemotherapy (AC) to CCRT. The role of induction chemotherapy has been 
explored, and the results of clinical trials of IC are also controversial. Three randomized Phase III trial from a NPCendemic area reported the encouraging result that addition of IC to CCRT significantly improved distant metastasisfree survival (DMFS) and OS in locoregionally advanced NPC. ${ }^{5-7}$ However, no benefit of survivals from induction chemotherapy adding to CCRT was observed in the other clinical trials. ${ }^{8,9}$ In consideration of these inconsistent clinical trial results, controversy exists regarding the value of IC in NPC. TPF is a category 1 option, but only for EBVassociated disease. ${ }^{3}$ Results from three systematic reviews had inconsistent results when evaluating the impact on survival outcomes, with two reviews showing that induction chemotherapy prior to systemic therapy/RT had superior OS and PFS rates, compared to systemic therapy/RT alone, ${ }^{10,11}$ while another review showed that induction chemotherapy prior to systemic therapy/RT did not have better survival outcomes than systemic therapy/RT alone or systemic therapy/RT followed by adjuvant chemotherapy. ${ }^{12}$ Expert groups (eg, ESMO, NCI) differ in their clinical practice guidelines regarding the use of induction chemotherapy for these patients, ${ }^{13}$ and the NCCN expert panel could not reach uniform consensus in this regard. Recent evidence has indicated that more than $75 \%$ of distant failures are concentrated in the advanced N-stage (stage N2-N3). ${ }^{14}$ Furthermore, high incidences of grade 3/4 adverse events were observed during the IC+CCRT groups. $^{5-7}$ So in the real world, the role of IC must be taken consideration, especially those patients with stage T1-2N1M0 or T3-4N0-1M0, which were excluded in the clinical trial. In clinical practice, these patients are considered to have a moderate risk of distant metastasis compared to stage T1-2N0M0 (low risk of metastasis) and stage $\mathrm{T}_{\text {any }}$ N2-3M0 (high risk of metastasis). Therefore, we performed this study to compare the clinical outcomes of patients with stage T1-2N1M0 and T3-4N0-1M0 NPC treated with IC followed by CCRT or CCRT alone.

\section{Materials and Methods}

\section{Patients}

Inclusion criteria were: (1) NPC confirmed histologically via nasopharyngeal biopsy; (2) no distant metastasis; (3) no prior treatment; (4) no previous malignancy or other concomitant malignant disease; (5) available pretreatment EBV DNA data, (6) restaged as T1-2N1M0 or T3-4N0$1 \mathrm{M} 0$ according to the 8th the American Joint Committee on Cancer (AJCC) staging system for $\mathrm{NPC}^{3}$ and (7) treatment with radical IMRT; 506 patients treated with IC plus CCRT or CCRT alone between 2005 and 2010 were included. Patients were excluded if their NPC was in stage T1-2N0M0 or $\mathrm{T}_{\text {any }} \mathrm{N} 2-3 \mathrm{M} 0$ or $\mathrm{T}_{\text {any }} \mathrm{N}_{\text {any }} \mathrm{M} 1$; if they had a second primary tumor or history of malignant tumors; if they were receiving adjuvant chemotherapy or radiotherapy alone; or if pretreatment EBV DNA data were unavailable. This retrospective study was approved by the Clinical Research Ethics Committee of Sun Yat-Sen University Cancer Canter. Patient records were anonymized prior to analysis. Informed consent was not required for this research due to the anonymized patient data.

Pretreatment evaluations included a detailed medical history, physical examination and performance status assessment. Routine workup included magnetic resonance imaging (MRI) of the nasopharynx and neck, chest radiograph, bone scintigraphy and abdominal ultrasonography for all patients. $20.1 \%(102 / 506)$ patients also underwent positron emission tomography/computed tomography (PET/CT) examination. The detection of EBV DNA copy was as previously described. ${ }^{15} 64.0 \%(324 / 506)$ patients had a positive EBV DNA copy.

\section{Treatment}

All patients received definitive IMRT with concurrent chemotherapy; $40.1 \%(203 / 506)$ received IC before concurrent chemoradiotherapy. The cumulative radiation doses were 68 Gy or greater to the primary tumor, $60-66$ Gy to involved cervical lymph nodes and 50 Gy or greater to potential sites of local infiltration and bilateral cervical lymphatics, in 30-33 fractions. The planning technique, target volume delineation and evaluation of IMRT planning were as previously described. ${ }^{4,16}$

The IC regimens included PF $(80 \mathrm{mg} / \mathrm{m} 2$ cisplatin on day 1 and $800 \mathrm{mg} / \mathrm{m} 2 / \mathrm{d}$ fluorouracil civ on days $1-5)$, TP $(75 \mathrm{mg} / \mathrm{m} 2$ docetaxel on day 1 and $75 \mathrm{mg} / \mathrm{m} 2$ cisplatin on day 1), or TPF $(60 \mathrm{mg} / \mathrm{m} 2$ docetaxel on day $1,60 \mathrm{mg} / \mathrm{m} 2$ cisplatin on day 1 , $600 \mathrm{mg} / \mathrm{m} 2 / \mathrm{d}$ fluorouracil civ on days $1-5$ ) for $2-3$ courses. Concurrent chemotherapy was mainly $80-100 \mathrm{mg} / \mathrm{m} 2$ of a cisplatin- or nedaplatin-based regimen given every three weeks for two to three cycles, or $30-40 \mathrm{mg} / \mathrm{m} 2$ of the cisplatin- or nedaplatin-based regimen or $20-30 \mathrm{mg} / \mathrm{m} 2$ of a docetaxel-based regimen given weekly for up to seven cycles. Patients who received only one cycle of IC or concurrent chemotherapy were excluded. In the IC group, $46.8 \%$ $(95 / 203)$ of patients received TPF, 30.5\% (62/203) received TP and 22.7\% (46/203) received PF. 


\section{Follow-Up}

All patients were followed up at regular intervals after completing treatment: every 3 months in the first 3 years, every 6 months for the next 2 years, then annually.

\section{Endpoints}

Study endpoints were OS, PFS, LRFS and DMFS. OS was defined the time from initiation of treatment to date of death from any cause or last follow-up; PFS to events that included death or disease progression at local, regional or distant sites or last follow-up; LRFS to primary site or neck lymph node relapse or last follow-up; DMFS to first observation of distant metastases or last follow-up.

\section{Statistical Analysis}

All statistical analyses were performed using SPSS version 22.0. Categorical variables were compared using the Chisquare test (or Fisher's exact test, if the expected number was less than five in at least $25 \%$ of cells). Survival rates were calculated using the Kaplan-Meier method and the Log-rank test was used to perform paired comparisons between treatment groups using the pair-wise over strata method. Receiver operation characteristic (ROC) curve was applied to calculate the cut-off value of pretreatment EBV DNA for DMFS. Multivariate analyses with the Cox proportional hazards model were used to calculate hazard ratios (HR), 95\% confidence intervals (CI) and test the independent significance of different factors. Two-tailed p-values $<0.05$ were considered statistically significant; $p$-value corrections for multiple comparisons were not performed.

\section{Results}

\section{Baseline Characteristics and Failure Patterns}

Table 1 summarizes the baseline clinical characteristics of the patients: 203 (40.1\%) patients received IC followed by CCRT and 303 (59.9\%) received CCRT alone. Patients with advanced clinical stage, advanced $\mathrm{T}$ category, or high EBV DNA copy number ( $\geq 4000$ copies $/ \mathrm{mL}$ ) were more likely to receive IC followed by CCRT (Table 1).

The median follow-up duration for the entire cohort was 69.3 months (range, 3.5-130.1 months). By last follow-up, 26 $(12.8 \%)$ patients in the IC + CCRT group and $29(9.6 \%)$ in the CCRT group had developed locoregional relapse, 22 (10.8\%) in the IC + CCRT group and $38(12.5 \%)$ in the CCRT group had developed distant metastasis, and $28(13.8 \%)$ in the IC + CCRT group and 43 (14.1\%) in the CCRT group had died.
Table I Baseline Characteristics of the Patients with Stage TI2NIM0 or T3-4N0-IMO Nasopharyngeal Carcinoma

\begin{tabular}{|c|c|c|c|}
\hline \multirow[t]{2}{*}{ Characteristic } & IC + CCRT & CCRT & $\mathbf{P}$ \\
\hline & $\begin{array}{l}n=203 \\
(40.1 \%)\end{array}$ & $\begin{array}{l}n=303 \\
(59.9 \%)\end{array}$ & \\
\hline $\begin{array}{l}\text { Gender } \\
\text { Male } \\
\text { Female }\end{array}$ & $\begin{array}{l}147(72.4 \%) \\
56(27.6 \%)\end{array}$ & $\begin{array}{l}208 \text { (68.6\%) } \\
95(31.4 \%)\end{array}$ & 0.364 \\
\hline $\begin{array}{l}\text { Age (years) } \\
\quad<45 \\
\geq 45\end{array}$ & $\begin{array}{l}\text { II } 4(56.2 \%) \\
89(43.8 \%)\end{array}$ & $\begin{array}{l}156(51.5 \%) \\
147(48.5 \%)\end{array}$ & 0.302 \\
\hline $\begin{array}{l}\text { T category } \\
\text { I } \\
2 \\
3 \\
4\end{array}$ & $\begin{array}{l}12(5.9 \%) \\
16(7.9 \%) \\
81(39.9 \%) \\
94(46.3 \%)\end{array}$ & $\begin{array}{l}27(8.9 \%) \\
49(16.2 \%) \\
154(50.8 \%) \\
73(24.1 \%)\end{array}$ & $<0.001$ \\
\hline $\begin{array}{c}\text { N category } \\
0 \\
\mathrm{I}\end{array}$ & $\begin{array}{l}3 \mid(15.3 \%) \\
172(84.7 \%)\end{array}$ & $\begin{array}{l}48(15.8 \%) \\
255(84.2 \%)\end{array}$ & 0.862 \\
\hline $\begin{array}{l}\text { Clinical stage } \\
\text { II } \\
\text { III } \\
\text { IV a }\end{array}$ & $\begin{array}{l}28(13.8 \%) \\
82(39.9 \%) \\
93(46.3 \%)\end{array}$ & $\begin{array}{l}78(25.1 \%) \\
152(50.8 \%) \\
73(24.1 \%)\end{array}$ & $<0.001$ \\
\hline $\begin{array}{l}\text { EBV DNA } \\
\quad<4000 \text { copies } / \mathrm{mL} \\
\quad \geq 4000 \text { copies } / \mathrm{mL}\end{array}$ & $\begin{array}{l}73(36.0 \%) \\
130(64.0 \%)\end{array}$ & $\begin{array}{l}186(61.4 \%) \\
117(38.6 \%)\end{array}$ & $<0.001$ \\
\hline
\end{tabular}

Abbreviations: IC, induction chemotherapy; CCRT, concurrent chemoradiotherapy; EBV DNA, Epstein-Barr virus deoxyribonucleic acid.

\section{Survival Outcomes}

The 5-year OS, PFS, locoregional relapse-free survival (LRFS), and DMFS rates for the entire cohort were $87.5 \%$, $79.6 \%, 88.8 \%$, and $88.7 \%$, respectively. Median follow-up duration was 70.2 months (range, 11.4-130.1 months) and 68.5 months (range, $3.5-112.6$ months) for the IC + CCRT and CCRT groups, respectively. The 5 -year OS rates for the IC + CCRT and CCRT groups were $88.5 \%$ and $86.8 \%$, respectively ( $p=0.661$, Figure 1A); the 5-year PFS rates were 79.6\% and $79.6 \%$ ( $p=0.756$, Figure 1B); the 5-year LRFS rates were $87.0 \%$ and $90.2 \%(p=0.364$, Figure $1 \mathrm{C})$, and the 5 -year DMFS rates were $89.8 \%$ and $88.0 \%$, respectively ( $p=0.407$, Figure 1D). There was no significant difference in OS, PFS, LRFS or DMFS between the IC + CCRT and CCRT groups.

Consistent with the results of univariate analysis, multivariate analysis to adjust for various potential prognostic factors revealed IC was not associated with significantly improved OS (HR 0.76, 95\% CI 0.46-1.25, $p=0.278$ ), 


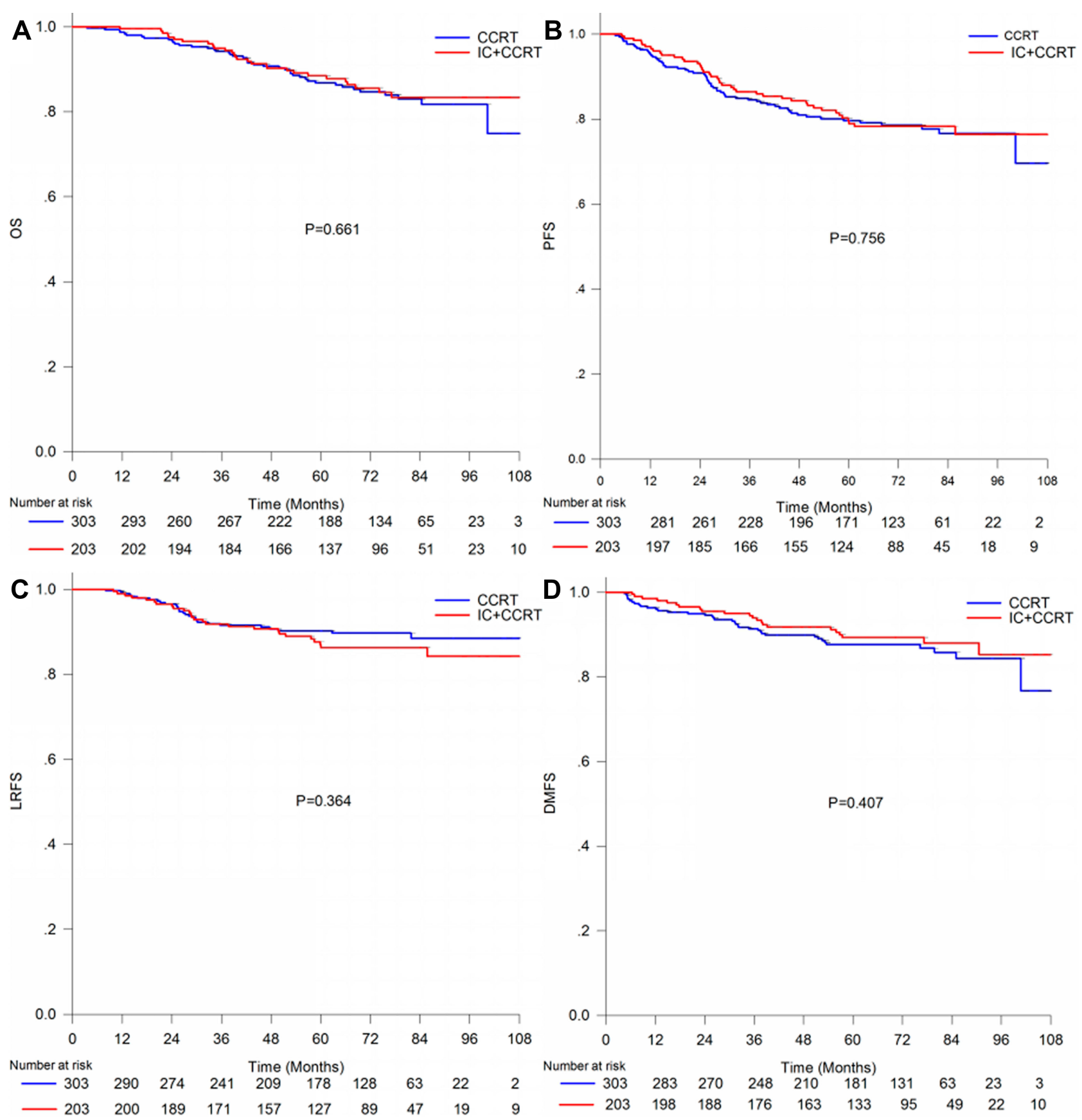

Figure I Kaplan-Meier survival curves for patients with stage TI-2NIM0 or T3-4N0-IM0 nasopharyngeal carcinoma treated with IC + CCRT or CCRT alone. (A) Overall survival; (B) progression-free survival; (C) locoregional relapse-free survival; (D) distant metastasis-free survival.

Abbreviations: IC, induction chemotherapy; CCRT, concurrent chemoradiotherapy; NPC, nasopharyngeal carcinoma.

PFS (HR 0.78, 95\% CI 0.51-1.19, $p=0.244)$, LRFS (HR $1.06,95 \%$ CI $0.81-1.42, p=0.651$ ) or DMFS (HR 0.66, $95 \%$ CI $0.38-1.15, p=0.140$; Table 2).

Prognostic Value of IC in N0 and NI Category NPC

Lymph node metastasis is associated with a significantly higher rate of distant metastasis compared to patients without lymph node metastasis. Therefore, we conducted subgroup analysis to further investigate the efficacy of IC in N0 and N1 category NPC: 79 (15.6\%) patients had N0 category NPC and $427(84.4 \%)$ patients had N1 category NPC.

In N1 category NPC, no significant differences in 5 -year OS (89.5\% vs $86.0 \%, p=0.587$, Figure $2 \mathrm{~A})$, DMFS $(89.1 \%$ vs $87.6 \%, p=0.494$, Figure $2 \mathrm{~B})$, PFS 
Table 2 Summary of Multivariable Analyses of Prognostic Factors

\begin{tabular}{|c|c|c|c|}
\hline Endpoint & Variable & HR (95\% Cl) & $P$ \\
\hline \multirow[t]{7}{*}{ OS } & Age* & $1.04(1.01-1.06)$ & 0.003 \\
\hline & Gender† & $1.12(0.67-1.86)$ & 0.660 \\
\hline & EBV DNA $\ddagger$ & $1.34(0.8 \mathrm{I}-2.22)$ & 0.253 \\
\hline & T3 vs TI-2 & $0.77(0.34-1.75)$ & 0.530 \\
\hline & T4 vs TI-2 & $1.62(0.72-3.61)$ & 0.243 \\
\hline & NI vs No & $1.09(0.57-2.07)$ & 0.794 \\
\hline & IC + CCRT vs CCRT & $0.76(0.46-1.25)$ & 0.278 \\
\hline \multirow[t]{7}{*}{ PFS } & Age* & $1.02(0.79-1.83)$ & 0.032 \\
\hline & Gendert & $1.20(0.79-1.83)$ & 0.387 \\
\hline & EBV DNA $\ddagger$ & $1.29(0.86-1.96)$ & 0.221 \\
\hline & T3 vs TI-2 & $0.81(0.44-1.50)$ & 0.498 \\
\hline & T4 vs TI-2 & $1.59(0.86-2.92)$ & 0.139 \\
\hline & $\mathrm{NI}$ vs NO & $0.88(0.52-1.47)$ & 0.619 \\
\hline & IC + CCRT vs CCRT & $0.78(0.51-1.19)$ & 0.244 \\
\hline \multirow[t]{7}{*}{ LRFS } & Age* & $1.00(0.97-1.03)$ & 0.917 \\
\hline & Gender $^{\dagger}$ & $\mathrm{I} .42(0.8 \mathrm{I}-2.48)$ & 0.219 \\
\hline & EBV DNA ${ }^{\ddagger}$ & $1.37(0.78-2.42)$ & 0.278 \\
\hline & T3 vs TI-2 & $0.47(0.22-1.00)$ & 0.050 \\
\hline & T4 vs TI-2 & $0.78(0.37-1.67)$ & 0.529 \\
\hline & $\mathrm{NI}$ vs NO & $0.66(0.32-1.32)$ & 0.237 \\
\hline & IC + CCRT vs CCRT & $1.06(0.81-1.42)$ & 0.651 \\
\hline \multirow[t]{7}{*}{ DMFS } & Age* & $1.01(0.98-1.04)$ & 0.340 \\
\hline & Gender $^{\dagger}$ & $0.66(0.35-1.23)$ & 0.190 \\
\hline & EBV DNA ${ }^{\ddagger}$ & $2.19(1.24-3.85)$ & 0.007 \\
\hline & T3 vs TI-2 & $0.75(0.37-1.52)$ & 0.427 \\
\hline & T4 vs TI-2 & $0.83(0.39-1.76)$ & 0.625 \\
\hline & $\mathrm{NI}$ vs No & $1.39(0.61-3.13)$ & 0.433 \\
\hline & IC + CCRT vs CCRT & $0.66(0.38-1.15)$ & 0.140 \\
\hline
\end{tabular}

Notes: $P$-values were calculated using an adjusted Cox proportional hazards model. *Age per year increase. ${ }^{\dagger} W o m e n$ vs men. ${ }^{\ddagger} \geq 4000$ vs $<4000$ copies $/ \mathrm{mL}$.

Abbreviations: $\mathrm{HR}$, hazard ratio; $\mathrm{Cl}$, confidence interval; EBV DNA: Epstein-Barr virus deoxyribonucleic acid; OS, overall survival; PFS, progression-free survival; LRFS, locoregional relapse-free survival; DMFS, distant metastasis-free survival; IC, induction chemotherapy; CCRT, concurrent chemoradiotherapy.

(79.3\% vs $80.5 \%, p=0.862)$ or LRFS $(87.7 \%$ vs $91.0 \%$, $p=0.394)$ were found between the IC + CCRT and CCRT groups.

Similarly, 5-year OS (83.9\% vs $87.4 \%, p=0.721$, Figure 2C), DMFS (93.5\% vs 89.6\%, $p=0.529$, Figure 2D), PFS (77.4\% vs $74.9 \%, p=0.715)$ and LRFS (83.6\% vs $86.5 \%$, $p=0.744)$ were comparable between the IC + CCRT and CCRT groups in N0 category NPC.

\section{Prognostic Value of IC in Patients with $\geq$ or $<4000$ EBV DNA Copies/mL}

Patients with $\geq 4000$ EBV DNA copies/mL generally exhibit a higher rate of distant metastasis than patients with $<4000$ EBV DNA copies/mL. ${ }^{17,18}$ The cut-off value of pretreatment
EBV DNA was 3995 copies/mL for DMFS. Therefore, we conducted stratified analysis of the prognostic value of IC among the 247 (48.8\%) patients with $\geq 4000$ copies $/ \mathrm{mL}$ and $259(51.2 \%)$ patients with $<4000$ copies $/ \mathrm{mL}$.

Among patients with $\geq 4000$ copies $/ \mathrm{mL}, 5$-year OS (88.7\% vs $82.8 \%, p=0.214$, Figure $3 \mathrm{~A})$, DMFS $(82.7 \%$ vs $88.1 \%, p=0.105$, Figure $3 \mathrm{~B}$ ), LRFS (83.9\% vs $90.4 \%$, $p=0.249)$ and PFS (78.0\% vs $75.8 \%, p=0.441)$ were comparable between patients receiving CCRT + IC and CCRT. Moreover, among patients with $<4000$ copies $/ \mathrm{mL}$, IC did not significantly improve 5-year OS $(88.0 \%$ vs 89.2.0\%, $p=0.822$, Figure 3C), DMFS (92.9\% vs $91.4 \%, p=0.719$, Figure 3D), LRFS (92.6\% vs $90.0 \%$, $p=0.692)$ or PFS $(82.4 \%$ vs $82.0 \%, p=0.891)$.

\section{Subgroup Analysis of the Prognostic Value of TPF IC}

In the latest published Phase III trial, TPF regimen IC significantly improved failure-free survival with acceptable toxicities in locally advanced NPC. ${ }^{6}$ Therefore, we compared the survival outcomes of patients receiving TPF regimen IC, other IC regimens and CCRT alone.

Five-year OS (89.1\% vs 86.8\%, $p=0.739)$, DMFS $(89.0 \%$ vs $88.0 \%, p=0.593)$, PFS $(77.6 \%$ vs $79.6 \%, p=0.995)$ and LRFS (82.4\% vs $90.2 \%, p=0.111$; Table 3 ) were comparable between patients receiving TPF regimen IC and patients receiving CCRT alone.

Five 5-year OS (88.2\% vs 86.8\%, $p=0.730)$, DMFS (90.5\% vs $88.0 \%, p=0.449)$, PFS $(81.4 \%$ vs $79.6 \%$, $p=0.631)$ and LRFS (91.0\% vs $90.2 \%, p=0.933)$ (Table 3) were also comparable between patients receiving IC based on the TP or PF regimens and patients receiving CCRT alone.

We also estimated the prognostic significance of IC for patients with T1-2N1M0 or T3-4N0-1M0 NPC. Compared to CCRT, IC + CCRT alone had no prognostic significance in T1-2N1M0 NPC ( $p=0.706$ for OS, $p=0.724$ for DMFS, $p=0.403$ for PFS, $p=0.371$ for LRFS) or T3-4N0-1M0 NPC $(p=0.486$ for OS, $p=0.309$ for DMFS, $p=0.422$ for PFS, $p=0.530$ for LRFS; Table 3 ).

\section{Discussion}

Recent clinical trials showed the benefit of IC in local advanced NPC, ${ }^{5-7}$ the results in the real world were controversial. Three Bayesian network meta-analyses have shown IC plus CCRT reduces distant metastasis but does not improve OS in NPC compared to CCRT alone. ${ }^{19-21}$ In 


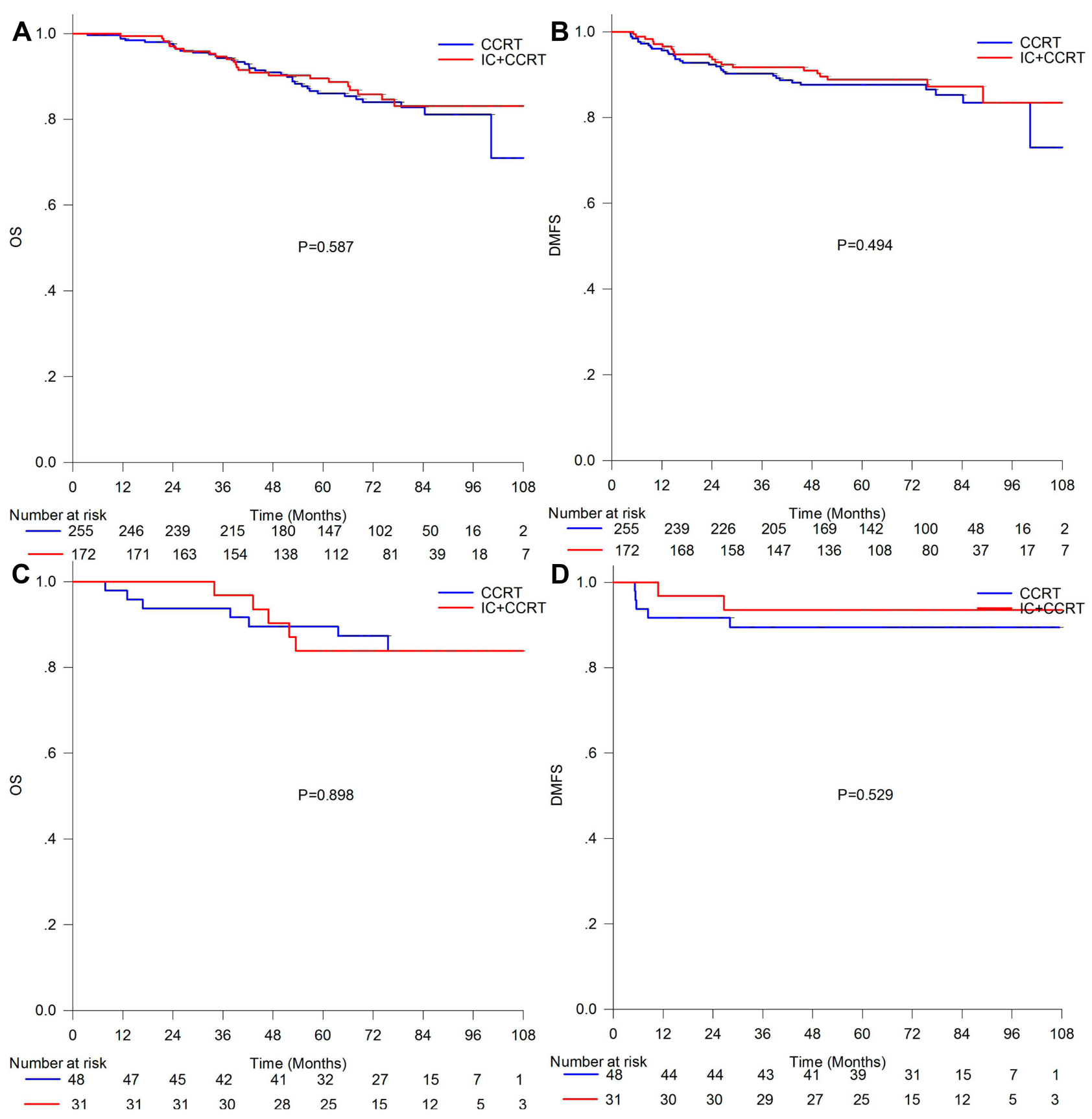

Figure 2 Kaplan-Meier survival curves for patients with stage TI-2NIM0 or T3-4N0-IM0 nasopharyngeal carcinoma treated with IC + CCRT or CCRT alone stratified by $\mathrm{N}$ category. (A and $\mathbf{C})$ Overall survival; (B and $\mathbf{D})$ distant metastasis-free survival.

Abbreviations: IC, induction chemotherapy; CCRT, concurrent chemoradiotherapy; NPC, nasopharyngeal carcinoma.

our study, we focus on those patients with stage T1-2N1M0 or T3-4N0-1M0 NPC, which mostly were excluded in RCT. We found that the addition of IC to radical CCRT did not lower the risk of death, progression, locoregional relapse or distant metastasis. Stratified analysis based on $\mathrm{N}$ category and EBV DNA further demonstrated IC did not improve the efficacy of CCRT in terms of reducing distant metastasis.
IC may not provide any value for patients at low risk of distant metastasis, and may even weaken the effect of radiotherapy. In the retrospective study of patients with stage II, IC caused deleterious effect and was a negative factor associated with OS (HR of death $=3.768,95 \% \mathrm{CI}=$ 1.117-12.709; $\mathrm{P}=0.032$ ), PFS (HR of progression $=$ 2.156, 95\% CI $=1.060-4.386 ; \mathrm{P}=0.034)$, LRFFS (HR of locoregional relapse $=2.435,95 \% \mathrm{CI}=1.009-5.874$; 


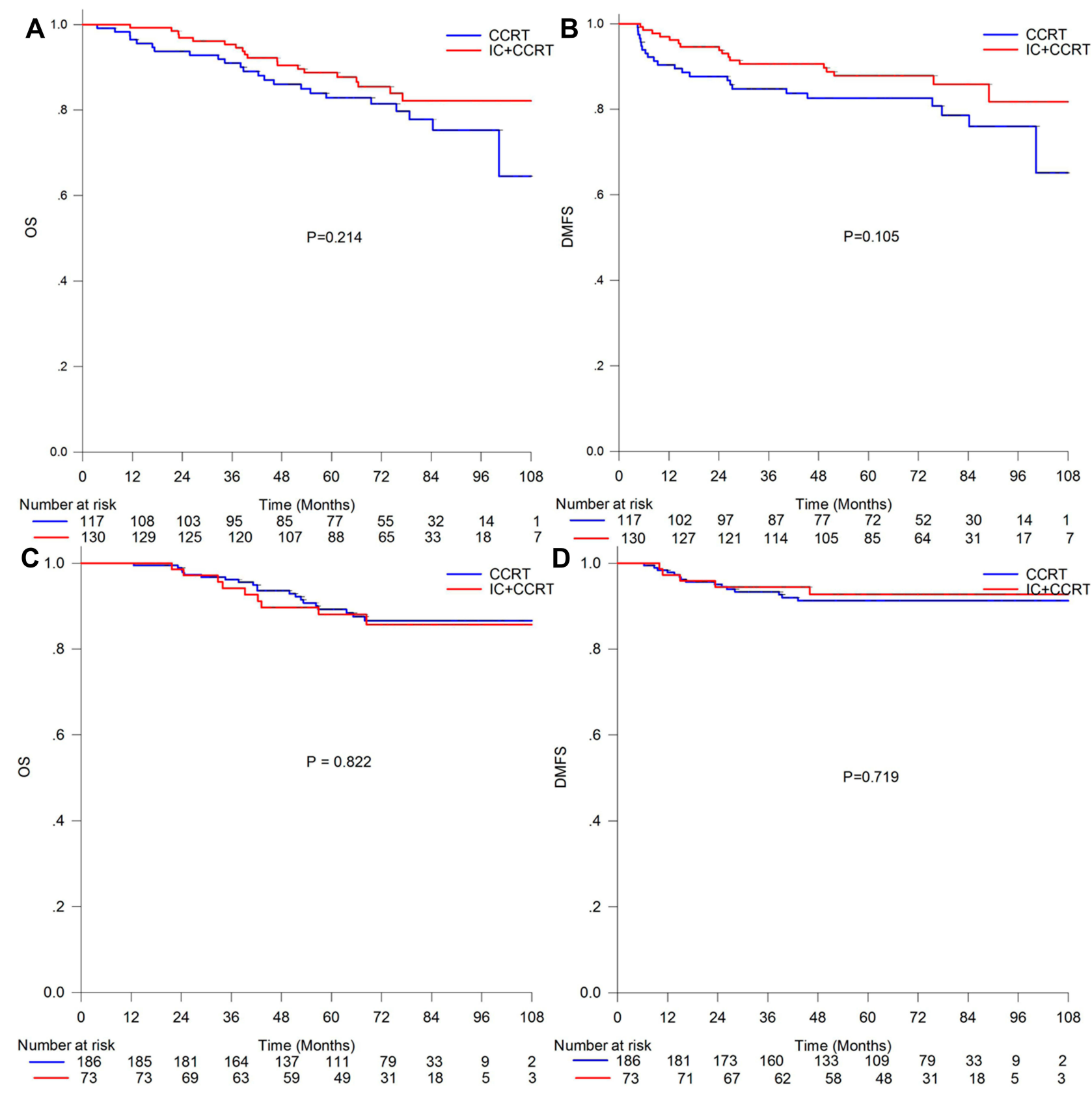

Figure 3 Kaplan-Meier survival curves for patients with stage TI-2NIM0 or T3-4N0-IM0 nasopharyngeal carcinoma treated with IC + CCRT or CCRT alone and stratified by EBV DNA copy number ( $\geq 4000$ vs $<4000$ copies $/ \mathrm{mL}$ ). (A and C) Overall survival; (B and $\mathbf{D})$ distant metastasis-free survival.

Abbreviations: IC, induction chemotherapy; CCRT, concurrent chemoradiotherapy; NPC, nasopharyngeal carcinoma; EBV DNA, Epstein-Barr virus deoxyribonucleic acid.

$\mathrm{P}=0.048$ ) and also DMFS (HR of metastasis $=2.873$, $95 \% \mathrm{CI}=1.005-8.211 ; \mathrm{P}=0.049) .{ }^{22} \mathrm{IC}$ was reported to improve DFS in stage IV N2-3 NPC compared with CCRT, indicating only selected patients benefit from IC. ${ }^{23}$ Advanced N category (N2-3) is well recognized as a risk factor for distant metastasis in the clinic; a clinical trial of patients with T1-4N2-3M0 is currently underway (NCT02512315). Patients with advanced N-stage (stage N2-3) NPC may not all can benefit from IC. No significant difference between IC plus CCRT and CCRT was observed ( $p=0.831$ and 0.608 , respectively) in the intermediate and low-risk groups. ${ }^{24}$ The results told us that careful consideration of IC must be taken, especially in those patients considered lower distant metastasis risk. In this study, we focused on stage T1-2N1M0 and T3-4N0-1M0 NPC. To identify the subgroups who may benefit from IC, we performed stratified analysis based on $\mathrm{N}$ category. However, IC provided no significant survival benefit in 
Table 3 Subgroup Analysis of the Prognostic Value of Induction Chemotherapy

\begin{tabular}{|c|c|c|c|c|c|c|c|c|}
\hline & \multicolumn{4}{|l|}{ os } & \multicolumn{4}{|l|}{ DMFS } \\
\hline & $\begin{array}{l}\text { Number of } \\
\text { Events }\end{array}$ & $\begin{array}{l}\text { Total } \\
\text { Number }\end{array}$ & $\begin{array}{l}5 \text {-Year } \\
\text { OS }\end{array}$ & $P$ & $\begin{array}{l}\text { Number of } \\
\text { Events }\end{array}$ & $\begin{array}{l}\text { Total } \\
\text { Number }\end{array}$ & $\begin{array}{l}\text { 5-Year } \\
\text { DMFS }\end{array}$ & $P$ \\
\hline \multicolumn{9}{|l|}{ TPF Regimen IC } \\
\hline IC + CCRT & 12 & 95 & $89.1 \%$ & 0.739 & 10 & 95 & $89.0 \%$ & 0.593 \\
\hline CCRT alone & 43 & 303 & $86.8 \%$ & & 38 & 303 & $88.0 \%$ & \\
\hline \multicolumn{9}{|l|}{ TP or PF Regimen IC } \\
\hline IC + CCRT & 16 & 108 & $88.2 \%$ & 0.720 & 12 & 108 & $90.5 \%$ & 0.449 \\
\hline CCRT alone & 43 & 303 & $86.8 \%$ & & 38 & 303 & $88.0 \%$ & \\
\hline \multicolumn{9}{|l|}{ TI-2NIMO } \\
\hline IC + CCRT & 3 & 28 & $91.6 \%$ & 0.706 & 4 & 28 & $82.9 \%$ & 0.724 \\
\hline CCRT alone & 5 & 76 & $86.3 \%$ & & 8 & 76 & $87.5 \%$ & \\
\hline \multicolumn{9}{|l|}{ T3-4NO-IMO } \\
\hline IC + CCRT & 25 & 175 & $88.2 \%$ & 0.486 & 18 & 175 & $90.6 \%$ & 0.309 \\
\hline CCRT alone & 38 & 227 & $86.1 \%$ & & 30 & 227 & $88.1 \%$ & \\
\hline
\end{tabular}

Notes: TPF was $60 \mathrm{mg} / \mathrm{m}^{2}$ docetaxel on day I, $60 \mathrm{mg} / \mathrm{m}^{2}$ cisplatin on day I and $600 \mathrm{mg} / \mathrm{m}^{2} / \mathrm{d}$ fluorouracil civ on days I-5; TP was $75 \mathrm{mg} / \mathrm{m}^{2}$ docetaxel on day I and $75 \mathrm{mg} / \mathrm{m}^{2}$ cisplatin on day I; PF was $80 \mathrm{mg} / \mathrm{m}^{2}$ cisplatin on day I and $800 \mathrm{mg} / \mathrm{m}^{2} / \mathrm{d}$ fluorouracil civ on days I-5. P-values were calculated using the Log-rank test.

Abbreviations: OS, overall survival; DMFS, distant metastasis-free survival; IC, induction chemotherapy; CCRT, concurrent chemoradiotherapy.

the subgroup analysis. These negative results could be attributed to three factors. Firstly, IC may not actually reduce distant metastasis. Secondly, the relatively small sample sizes in each subgroup may have resulted in low statistical power, so differences between subgroups could not be detected. Third, several large-scale Phase III trials have confirmed adding docetaxel to PF regimen IC provides a significant clinical benefit. ${ }^{25-27}$ However, a largescale propensity-matched study indicated taxane-based IC could not improve survival in locoregionally advanced NPC, but decreased the risk of distant metastasis in stage T4N1-2M0 and stage IVb NPC. ${ }^{28}$ In this study, we only assessed patients with stage T1-2N1M0 and T3-4N0-1M0 NPC; $46.8 \%$ of patients received TPF and $22.7 \%$ received PF. The use of different IC chemotherapy regimens in this cohort may provide a slight bias towards a negative result. However, subgroup analysis was used to reduce the potential bias associated with different chemotherapy regimens, and demonstrated the TPF regimen did not have any significant prognostic value. Further studies are required to verify these results. Finally, more cycles of IC might be essential. Four cycles of IC were effective and well tolerated. ${ }^{29} \mathrm{~A}$ trial on 4 cycles of neoadjuvant chemotherapy plus concurrent chemoradiation in N2-3 nasopharyngeal carcinoma is ongoing (NCT02512315).

EBV DNA is the most effective prognostic biomarker for guiding the treatment of NPC. High pre-treatment EBV
DNA can accurately predict distant metastasis. ${ }^{15,30}$ Detectable EBV DNA is associated with poorer DMFS compared to undetectable EBV DNA. ${ }^{31}$ IC was previously reported to improve the survival of patients with high EBV DNA. ${ }^{32}$ In our study, EBV DNA ( $\geq 4000$ vs $<4000$ copies/ $\mathrm{mL}$ ) was an independent prognostic factor for DMFS (HR $2.19,95 \%$ CI $1.24-3.85, p=0.007)$. However, IC did not provide a significant survival benefit in stratified analysis of patients with $\geq 4000$ or $<4000$ EBV DNA copies $/ \mathrm{mL}$, which might due to the unbalanced patients. In our enrolled patients, $64 \%$ of patients who received IC had EBV DNA $\geq 4000$ copies $/ \mathrm{mL}$, while CCRT was $38 \%$. In clinical, Patients with high EBV NDA copies might be treated with greater intensity treatment. However, a propensity-matched analysis of stage III-IVb patients with $\geq 4000$ EBV DNA copies $/ \mathrm{mL}$ showed IC did not improve the clinical outcomes of these high-risk patients compared to CCRT alone, while IC only improved OS in very high-risk patients (N2-3 category with EBV DNA $\geq 4000$ copies $/ \mathrm{mL}) .{ }^{17}$ Hence, the use of IC should be carefully considered in patients with stage T1-2N1M0 and T3-4N0-1M0, even though some of these patients have a high EBV DNA copy number. Further prospective studies are needed to more precisely evaluate the role of EBV DNA for guiding the treatment of different subgroups of patients with NPC. A clinical trial of individualized treatment of patients with stage II-IVB NPC based on EBV DNA is currently ongoing (NCT02135042). 
The exploration on IC for local advanced NPC is still ongoing. Whether benefit of IC is uncertain. The incidences of toxicity (a higher risk of neutropenia and weight loss, and so on) were increased proved by the clinical trials. ${ }^{5-8}$ Other than improving distant metastasis control, one of the targets of IC in clinical for oncologist might be to shrink the tumor volume to reduce the side reaction of radiation therapy. A lower risk of mucositis and hearing loss was found in IC+CCRT compared to CCRT. ${ }^{12}$ The proportion of patients with eye damage was significantly lower in the IC followed by CCRT alone group than the CCRT group $(9.7 \%$ versus $16.4 \%, \mathrm{p}=0.029) .{ }^{5}$ Furthermore, an interesting finding from Taiwan which the patients with stage N2-3 accounting $85 \%$ showed that IC significantly improved the LRFS $(\mathrm{HR}=0.74$, $\mathrm{p}=0.026)$ and failed to reduce distant metastasis $(\mathrm{HR}=0.82$, $\mathrm{p}=0.2828) .^{23}$ So selecting patients with nasopharyngeal carcinoma who benefit from induction chemotherapy was important. A prognostic nomograms based on 8th edition of the UICC staging system found that IC could only improve OS for patients with stage III-IV at intermediate to high risks (including Histological type, T category, N category, plasma Epstein-Barr virus deoxyribonucleic acid, age and neutrophil-lymphocyte ratio). ${ }^{33}$ In the past few years, researchers and we focused on the clinical characteristics to select patients who can benefit from IC. More and more attentions are paid on the immune molecules and the immune system. Increased tumour-infiltrating lymphocytes (TIL) concentration predicted response to neoadjuvant chemotherapy in breast cancer, and was also associated with a survival benefit in HER2-positive breast cancer and TNBC. ${ }^{34}$ Infiltrating lymphocytes (IL)17Ap cells infiltration could be used as an independent prognostic biomarker for OS and predictive biomarker for superior response to adjuvant chemotherapy. ${ }^{35}$ Stromal immunotype predict survival and benefit from adjuvant chemotherapy in patients with muscle-invasive bladder cancer. ${ }^{36}$ TIL, immunotype might predict the response to chemotherapy. In the future, we might need to combine clinical characteristics and immune molecules and the immune system to select NPC patient who can benefit from IC. Further research investigating is warranted.

There are some limitations that must be noted. First, we just focused on the clinical characteristics to select patients who can benefit from IC. In future study, we should pay attention to molecular markers, such as TIL, immune classification and so on. Another limitation was the heterogeneity of the IC regimen and dosage because of the retrospective study design. Finally, the data on cute and late toxicities, treatment-related toxicities were lack due to our failing to collect in our intelligence platform in the current study.

In conclusion, IC + CCRT did not improve any survival outcome in patients with stage T1-2N1M0 or T3-4N0-1M0 NPC receiving IMRT compared with CCRT alone. IC has limited prognostic value in moderate-risk NPC, and should not be routinely prescribed in clinical practice to patients with T1-2N1M0 and T3-4N0-1M0 NPC. However, as this was a retrospective single-center study, prospective studies are warranted to confirm the value of IC in moderate-risk NPC.

\section{Abbreviations}

NPC, nasopharyngeal carcinoma; OS, overall survival; CCRT, concurrent chemoradiotherapy; NCCN, National Comprehensive Cancer Network; IMRT, intensitymodulated radiation therapy; IC, induction chemotherapy; AC, adjuvant chemotherapy; PFS, progression-free survival; DMFS, distant metastasis-free survival; EBV DNA, EpsteinBarr virus deoxyribonucleic acid; AJCC, American Joint Committee on Cancer; MRI, magnetic resonance imaging; LRFS, locoregional relapse-free survival; PET/CT, positron emission tomography/computed tomography; ROC, receiver operation characteristic; HR, hazard ratios; CI, confidence intervals.

\section{Data Sharing Statement}

All data generated or analyzed during the current study are available from the corresponding author upon reasonable request.

\section{Ethics Statement}

This retrospective study was approved by the Clinical Research Ethics Committee of Sun Yat-Sen University Cancer Canter. All procedures performed in studies involving human participants were in accordance with the ethical standards of the institutional and/or national research committee and with the 1964 Helsinki Declaration and its later amendments or comparable ethical standards.

\section{Informed Consent}

Informed consent was not required for this research due to the anonymized patient data.

\section{Acknowledgments}

We appreciated all individuals, particularly the people of Clinical Research Ethics Committee of Sun Yat-Sen University Cancer Center, including doctors, nurses, 
coordinators, and the Data Management team who generously spared their time for the accomplishment and fulfillment of this project.

\section{Author Contributions}

ZS, GRZ, JT, XYL and FYX contributed to conception and design of the study; ZS, GRZ, JT, and XYL contributed to acquisition of data, or analysis and interpretation of data; ZS, GRZ, JT, and XYL contributed to drafting the article. ZS, GRZ, and FYX contributed to revising the article critically for important intellectual content. ZS, GRZ, JT, XYL and FYX gave final approval of the version to be published, and agreement to be accountable for all aspects of the work in ensuring that questions related to the accuracy or integrity of any part of the work are appropriately investigated and resolved.

\section{Funding}

This research did not receive any specific grant from funding agencies in the public, commercial, or not-forprofit sectors.

\section{Disclosure}

The authors have no competing interests to declare.

\section{References}

1. Bray F, Ferlay J, Soerjomataram I, Siegel RL, Torre LA, Jemal A. Global cancer statistics 2018: GLOBOCAN estimates of incidence and mortality worldwide for 36 cancers in 185 countries. CA Cancer J Clin. 2018;68(6):394-424. doi:10.3322/caac.v68.6

2. Wee JT, Ha TC, Loong SL, Qian CN. Is nasopharyngeal cancer really a "Cantonese cancer"? Chin J Cancer. 2010;29(5):517-526. doi:10.5732/ cjc.009.10329

3. Pfister DG, Spencer S, Adelstein D, et al. Head and neck cancers. Natl Compr Cancer Net. 2019.

4. Sun X, Su S, Chen C, et al. Long-term outcomes of intensity-modulated radiotherapy for 868 patients with nasopharyngeal carcinoma: an analysis of survival and treatment toxicities. Radiother Oncol. 2014;110 (3):398-403. doi:10.1016/j.radonc.2013.10.020

5. Yang Q, Cao SM, Guo L, et al. Induction chemotherapy followed by concurrent chemoradiotherapy versus concurrent chemoradiotherapy alone in locoregionally advanced nasopharyngeal carcinoma: long-term results of a phase III multicentre randomised controlled trial. Eur J Cancer. 2019;119:87-96. doi:10.1016/j.ejca.2019.07.007

6. Sun Y, Li W, Chen N, et al. Induction chemotherapy plus concurrent chemoradiotherapy versus concurrent chemoradiotherapy alone in locoregionally advanced nasopharyngeal carcinoma: a Phase 3, multicentre, randomised controlled trial. Lancet Oncol. 2016;17 (11):1509-1520. doi:10.1016/S1470-2045(16)30410-7

7. Zhang Y, Chen L, Hu G, et al. Gemcitabine and cisplatin induction chemotherapy in nasopharyngeal carcinoma. New Engl J Med. 2019;381:1124-1135. doi:10.1056/NEJMoa1905287

8. Tan T, Lim WT, Fong KW, et al. Concurrent chemo-radiation with or without induction gemcitabine, carboplatin, and paclitaxel: a randomized, Phase $2 / 3$ trial in locally advanced nasopharyngeal carcinoma. Int J Radiat Oncol Biol Phys. 2015;91(5):952-960. doi:10.1016/j. ijrobp.2015.01.002
9. Fountzilas G, Ciuleanu E, Bobos M, et al. Induction chemotherapy followed by concomitant radiotherapy and weekly cisplatin versus the same concomitant chemoradiotherapy in patients with nasopharyngeal carcinoma: a randomized Phase II study conducted by the Hellenic Cooperative Oncology Group (HeCOG) with biomarker evaluation. Ann Oncol. 2012;23(2):427-435. doi:10.1093/annonc/ mdr116

10. Chen YP, Tang LL, Yang Q, et al. Induction chemotherapy plus concurrent chemoradiotherapy in endemic nasopharyngeal carcinoma: individual patient data pooled analysis of four randomized trials. Clin Cancer Res. 2018;24(8):1824-1833. doi:10.1158/10780432.CCR-17-2656

11. Tan TH, Soon YY, Cheo T, et al. Induction chemotherapy for locally advanced nasopharyngeal carcinoma treated with concurrent chemoradiation: a systematic review and meta-analysis. Radiother Oncol. 2018;129(1):10-17. doi:10.1016/j.radonc.2018.02.027

12. Ribassin-majed L, Marguet S, Lee A, et al. What is the best treatment of locally advanced nasopharyngeal carcinoma? An individual patient data network meta-analysis. J Clin Oncol. 2017;35(5):498-505. doi:10.1200/JCO.2016.67.4119

13. Chen Y-P, Wang Y-Q, Li W-F, et al. Critical evaluation of the quality and recommendations of clinical practice guidelines for nasopharyngeal carcinoma. J Natl Compr Canc Netw. 2017;15(3):336-344. doi:10.6004/jncen.2017.0033

14. Mao Y-P, Tang -L-L, Chen L, et al. Prognostic factors and failure patterns in non-metastatic nasopharyngeal carcinoma after intensity-modulated radiotherapy. Chin J Cancer. 2016;35(1):103. doi:10.1186/s40880-016-0167-2

15. Peng H, Guo R, Chen L, et al. Prognostic impact of plasma Epstein-Barr virus DNA in patients with nasopharyngeal carcinoma treated using intensity-modulated radiation therapy. Sci Rep. 2016;6:22000. doi:10.1038/srep22000

16. Su S, Han F, Zhao C, et al. Long-term outcomes of early-stage nasopharyngeal carcinoma patients treated with intensity-modulated radiotherapy alone. Int J Rad Oncol Biol Phys. 2012;82(1):327-333. doi:10.1016/j.ijrobp.2010.09.011

17. Guo SS, Tang LQ, Chen QY, et al. Induction chemotherapy followed by concurrent chemoradiotherapy versus concurrent chemoradiotherapy alone in stage III-IVb nasopharyngeal carcinoma patients with Epstein-Barr virus DNA $>/=4000$ copies $/ \mathrm{mL}$ : a matched study. Oncotarget. 2016;7(20):29739.

18. Du X-J, Tang L-L, Chen L, et al. Neoadjuvant chemotherapy in locally advanced nasopharyngeal carcinoma: defining high-risk patients who may benefit before concurrent chemotherapy combined with intensity-modulated radiotherapy. Sci Rep. 2015;5:16664. doi:10.1038/srep16664

19. Chen Y, Guo R, Liu N, et al. Efficacy of the additional neoadjuvant chemotherapy to concurrent chemoradiotherapy for patients with locoregionally advanced nasopharyngeal carcinoma: a Bayesian network meta-analysis of randomized controlled trials. J Cancer. 2015;6 (9):883-892. doi:10.7150/jca.11814

20. Chen YP, Wang ZX, Chen L, et al. A Bayesian network meta-analysis comparing concurrent chemoradiotherapy followed by adjuvant chemotherapy, concurrent chemoradiotherapy alone and radiotherapy alone in patients with locoregionally advanced nasopharyngeal carcinoma. Ann Oncol. 2015;26(1):205-211. doi:10.1093/annonc/ mdu507

21. Blanchard P, Lee A, Marguet S, et al. Chemotherapy and radiotherapy in nasopharyngeal carcinoma: an update of the MAC-NPC meta-analysis. Lancet Oncol. 2015;16(6):645-655. doi:10.1016/ S1470-2045(15)70126-9

22. Li P, Mo H, Luo D, Hu W, Jin T. The efficacy of induction chemotherapy in the treatment of stage II nasopharyngeal carcinoma in intensity modulated radiotherapy era. Oral Oncol. 2018;85:95-100. doi:10.1016/j.oraloncology.2018.08.016 
23. Hong RL, Hsiao CF, Ting LL, et al. Final results of a randomized phase III trial of induction chemotherapy followed by concurrent chemoradiotherapy versus concurrent chemoradiotherapy alone in patients with stage IVA and IVB nasopharyngeal carcinoma-Taiwan Cooperative Oncology Group (TCOG) 1303 Study. Ann Oncol. 2018;29(9):1972-1979. doi:10.1093/annonc/mdy249

24. Yao J, Jin Y, Liu Z, et al. Do all patients with advanced N-stage nasopharyngeal carcinoma benefit from the addition of induction chemotherapy to concurrent chemoradiotherapy? Ther Adv Med Oncol. 2019;11:386244890. doi:10.1177/1758835919833863

25. Vermorken JB, Remenar E, van Herpen C, et al. Cisplatin, fluorouracil, and docetaxel in unresectable head and neck cancer. $N$ Engl $J$ Med. 2007;357(17):1695-1704. doi:10.1056/NEJMoa071028

26. Posner MR, Hershock DM, Blajman CR, et al. Cisplatin and fluorouracil alone or with docetaxel in head and neck cancer. $N$ Engl $J$ Med. 2007;357(17):1705-1715. doi:10.1056/NEJMoa070956

27. Pointreau Y, Garaud P, Chapet S, et al. Randomized trial of induction chemotherapy with cisplatin and 5-fluorouracil with or without docetaxel for larynx preservation. J Natl Cancer Inst. 2009;101 (7):498-506. doi:10.1093/jnci/djp007

28. Zhang LN, Gao YH, Lan XW, Tang J, OuYang PY, Xie FY. Effect of taxanes-based induction chemotherapy in locoregionally advanced nasopharyngeal carcinoma: a large scale propensity-matched study. Oral Oncol. 2015;51(10):950-956. doi:10.1016/j.oraloncology.2015.07.004

29. Zhang Y, Chen M, Chen C, Kong L, Lu JJ, Xu B. The efficacy and toxicities of intensive induction chemotherapy followed by concurrent chemoradiotherapy in nasopharyngeal carcinoma patients with N3 disease. Sci Rep-Uk. 2017;7:1.

30. Tang LQ, Chen QY, Fan W, et al. Prospective study of tailoring whole-body dual-modality [18F]fluorodeoxyglucose positron emission tomography/computed tomography with plasma Epstein-Barr virus DNA for detecting distant metastasis in endemic nasopharyngeal carcinoma at initial staging. J Clin Oncol. 2013;31(23):2861-2869. doi:10.1200/JCO.2012.46.0816
31. Peng H, Chen L, Zhang Y, et al. Survival analysis of patients with advanced-stage nasopharyngeal carcinoma according to the Epstein-Barr virus status. Oncotarget. 2016;7(17):24208-24216. doi:10.18632/oncotarget.v7i17

32. Zhang J, Peng H, Li W, et al. Individualized induction chemotherapy by pre-treatment plasma Epstein-Barr viral DNA in advanced nasopharyngeal carcinoma. BMC Cancer. 2018;18:1. doi:10.1186/s12885018-5177-9

33. $\mathrm{Xu} \mathrm{C}$, Chen $\mathrm{Y}$, Liu $\mathrm{X}$, et al. Establishing and applying nomograms based on the 8th edition of the UICC/AJCC staging system to select patients with nasopharyngeal carcinoma who benefit from induction chemotherapy plus concurrent chemoradiotherapy. Oral Oncol. 2017;69:99-107. doi:10.1016/j. oraloncology.2017.04.015

34. Denkert C, von Minckwitz G, Darb-esfahani S, et al. Tumourinfiltrating lymphocytes and prognosis in different subtypes of breast cancer: a pooled analysis of 3771 patients treated with neoadjuvant therapy. Lancet Oncol. 2018;19(1):40-50. doi:10.1016/S14702045(17)30904-X

35. Wang JT, Li H, Zhang H, et al. Intratumoral IL17-producing cells infiltration correlate with antitumor immune contexture and improved response to adjuvant chemotherapy in gastric cancer. Ann Oncol. 2019;30(2):266-273. doi:10.1093/annonc/mdy505

36. Fu H, Zhu Y, Wang Y, et al. Identification and validation of stromal immunotype predict survival and benefit from adjuvant chemotherapy in patients with muscle-invasive bladder cancer. Clin Cancer Res. 2018;24(13):3069-3078. doi:10.1158/10780432.CCR-17-2687
Therapeutics and Clinical Risk Management

\section{Publish your work in this journal}

Therapeutics and Clinical Risk Management is an international, peerreviewed journal of clinical therapeutics and risk management, focusing on concise rapid reporting of clinical studies in all therapeutic areas, outcomes, safety, and programs for the effective, safe, and sustained use of medicines. This journal is indexed on PubMed Central, CAS,

\section{Dovepress}

EMBase, Scopus and the Elsevier Bibliographic databases. The manuscript management system is completely online and includes a very quick and fair peer-review system, which is all easy to use. Visit http://www.dovepress.com/testimonials.php to read real quotes from published authors. 